\title{
Effect of Headache-Free Days on Disability, Productivity, Quality of Life, and Costs Among Individuals with Migraine
}

\author{
Richard B. Lipton, MD; Lulu Lee, PhD; Nicolas P. Saikali, MD, FAHS; Jvawnna Bell, MPH, MBA; \\ and Joshua M. Cohen, MD, MPH, FAHS
}

\begin{abstract}
BACKGROUND: The functional impairment associated with migraine can cause physical, emotional, and economic ramifications that can affect occupational, academic, social, and family life. Understanding the relationship between headache-free days (HFDs) and the disease burden of migraine may help with decisions regarding treatment and management of migraine.

OBJECTIVE: To determine the relationship between burden of disease measures and HFDs among individuals with migraine experiencing $\geq 4$ headache days in the previous 30 days.

METHODS: The 2016 U.S. National Health and Wellness Survey $(\mathrm{N}=97,503)$ was self-administered to a nationally representative sample of adults. Respondents with a migraine diagnosis who reported $\geq 4$ headache days a month were included in the analysis. The primary independent variable was the number of HFDs assessed as both a continuous (HFDs in the previous 30 days) and categorical ( $0-10,11-20$, and 21-26 HFDs) measure. HFDs were used to predict outcomes using separate generalized linear models. Outcomes included effect on functional status and well-being, measured by the 6-item Headache Impact Test (HIT-6) score; number of days of work and/or household activities missed due to migraine; annualized indirect costs due to work productivity loss (assessed via the Work Productivity and Activity Impairment questionnaire); and annualized direct costs due to health care resource use (health care provider visits, emergency room visits, and hospitalizations).
\end{abstract}

RESULTS: The survey included 372 respondents with diagnosed migraine and $\geq 4$ headache days per month. Using HFDs as a continuous variable, each additional HFD was associated with a 0.15 -point reduction in HIT-6 scores, a $5 \%$ reduction in both number of work days and household activities missed, and a $4 \%$ reduction in indirect costs; thus, a 5-day increase in HFDs would lead to a 0.75 -point reduction in HIT-6 scores, $25 \%$ reduction in days of work or household activities missed, and $20 \%$ reduction in indirect costs. Analyzing HFDs as a categorical variable, respondents experiencing 21-26 HFDs had lower HIT-6 total scores than those with $0-10$ HFDs (adjusted means: 66.59 vs. $63.91 ; P=0.001$ ) or those with $11-20$ HFDs (65.66 vs. $63.91, P=0.015$ ). Respondents experiencing 21-26 HFDs missed fewer work days than those with 0-10 HFDs (4.44 vs. $1.46, P=0.002)$ or those with $11-20$ HFDs (3.36 vs. $1.46, P=0.009$ ). Similarly, respondents with $11-20$ HFDs $(22.99$ vs. $9.72, P<0.001)$ and those with $21-26$ HFDs ( 22.99 vs. $7.34, P=0.001)$ were associated with fewer days of household activities missed due to migraine compared with respondents with 0-10 HFDs. Respondents with 21-26 HFDs per month had significantly lower indirect costs $(\$ 16,975$ vs. $\$ 6,919, P=0.025)$ than those with 0-10 HFDs.

CONCLUSIONS: A higher number of HFDs is associated with decreased headache-related disability among those with migraine. Interventions that increase the total number of HFDs may reduce the burden and cost associated with migraine.

J Manag Care Spec Pharm. 2020;26(10):1344-52

Copyright $\odot 2020$, Academy of Managed Care Pharmacy. All rights reserved.

\section{What is already known about this subject}

The functional impairment associated with migraine has physical, emotional, and economic ramifications that commonly affect occupational, academic, social, and family life.

Individuals with migraine have significantly lower health-related quality of life than those in the general population with no chronic conditions, and their employers incur substantial indirect costs.

\section{What this study adds}

Each incremental increase in headache-free days (HFDs) is associated with a reduction in headache-related adverse effect on functional status and well-being, a reduction in days of work and household activities missed due to migraine, and a reduction in indirect costs.

Increasing the number of HFDs may reduce the burden and cost associated with migraine.

$\mathrm{M}$ igraine is a debilitating disease that affects more than 1 billion individuals worldwide, and based on the Global Burden of Disease Study 2017, is the secondleading cause of years lived with disability after low back pain. ${ }^{1}$ The substantial functional impairment associated with migraine has physical, emotional, and economic ramifications that commonly affect occupational, academic, social, and family life. In general, individuals with migraine have significantly lower health-related quality of life (HRQOL) than those in the general population with no chronic conditions, while their employers incur substantial indirect costs. ${ }^{2}$ The total annual cost of migraine in the United States, based on cost estimates per person and prevalence estimates from the National Health and Nutrition Examination Survey, is estimated to be as high as $\$ 78$ billion (in 2014 U.S. dollars). ${ }^{3}$

Migraine can be categorized as episodic migraine (EM; characterized by $<15$ headache days per month) or chronic migraine (CM; characterized by $\geq 15$ headache days per month). In addition to variation in frequency, sufferers may also experience different intensities and durations of attacks along with a range of associated symptoms, leading to variable levels of functional impairment., ${ }^{4,5}$ Observational studies that assessed migraine-specific quality of life, disability, and productivity have shown significant disability associated with 
both EM and CM. ${ }^{6-8}$ Unsurprisingly, $\mathrm{CM}$ is associated with greater disease burden and higher total annual costs. ${ }^{3,6-8}$

Individuals with EM progress to $\mathrm{CM}$ at an annual rate of approximately $2.5 \%^{4}$; identifying those at risk of progression and implementing appropriate interventions could help reduce progression to $\mathrm{CM}$ and thus the overall burden of disease. While some risk factors are not readily modifiable (e.g., age, female sex, white race, lower educational level/socioeconomic status, and marital status), others (e.g., obesity, depression, acute headache medication overuse, ineffective acute treatment, caffeine intake, $\geq 4$ headache days per month) may be targeted through behavioral changes and pharmacological treatment. ${ }^{4,9,10}$

Given that headache frequency and medication overuse are among the risk factors for progression to $\mathrm{CM}$, the use of migraine preventive medication to reduce the frequency of headaches and increase the number of headache-free days (HFDs) might help to reduce the risk of progression. ${ }^{11}$ Guidelines developed by the American Headache Society recommend offering preventive treatment to patients experiencing either $\geq 3$ headache days per month and severe disability, $\geq 4$ headache days per month and some disability, or $\geq 6$ headache days per month regardless of the degree of disability.12 An analysis of the American Migraine Prevalence and Prevention study demonstrated using regression models that the fully adjusted odds of new-onset $\mathrm{CM}$ in participants with $\geq 5$ headache days per month were 5.1 times that of participants with 4 or fewer days per month. ${ }^{10}$ Although it has been suggested that 38\%-39\% of individuals with migraine should be offered or considered for preventive treatment, a 2007 study showed that only 12\%-13\% of people with migraine in the population used a preventive medication, indicating that a sizable proportion of those who might benefit from preventive treatment did not receive it. ${ }^{13}$

The objective of this study was to determine the relationship between HFDs and the burden of disease among individuals with $\mathrm{CM}$ and those with EM at risk of developing $\mathrm{CM}$ ( $\geq 4$ headache days in the past month). Our expectations were that as HFDs increase, the measures of effect, disability, and cost would decrease. These findings may help physicians and their patients with migraine make more informed decisions regarding treatment and management of migraine.

\section{Methods}

\section{Study Design and Data Source}

This was a noninterventional study based on data obtained from Kantar Health's cross-sectional 2016 U.S. National Health and Wellness Survey (NHWS). The NHWS is a selfadministered, internet-based questionnaire from a nationwide sample of adults (aged $\geq 18$ years; $N=97,503$ ). Potential respondents were recruited through an existing, general-purpose (not health care-specific), web-based consumer panel that recruits its members through opt-in emails, coregistration with panel partners, e-newsletter campaigns, banner placements, and affiliate networks. All panelists completed an in-depth demographic registration profile. The composition of the study panel sample was matched to the demographic composition of the U.S. population. The 2016 NHWS was granted exemption from review by the Pearl Institutional Review Board (Indianapolis, IN).

The NHWS base survey component was completed by all respondents to assess demographics, diseases experienced and diagnosed, and health outcomes. To reduce respondent fatigue (and maintain an average 30-minute survey across respondents), only a random subset of patients eligible to answer specific disease module (e.g., migraine) questions responded to those questions. This randomization was based on a priori-assigned probability. So, for example, a respondent with hypertension (assigned probability 10\%) and hepatitis C (90\%) would be much more likely to answer hepatitis C questions than hypertension questions, though selection is random in both cases (due to change, a respondent could be selected to answer one, both, or neither). The assigned probabilities generally coincided with the inverse of prevalence of a condition (i.e., more prevalent conditions were assigned lower probabilities and vice versa) to maximize sample size for less prevalent diseases. This general approach has been used in previous academic research. ${ }^{14}$ To be included in the migraine module, respondents had to indicate they were diagnosed with migraine by a health care professional, had to be randomly selected into the migraine module, and had to agree to participate. Respondents in the migraine module who reported experiencing $\geq 4$ headache days in the previous 30 days were included in the analyses of this study.

\section{Measures}

Demographics (e.g., age, gender, ethnicity) and health characteristics (e.g., smoking status, alcohol use, comorbidities, Charlson Comorbidity Index [CCI]) were evaluated along with migraine-related variables, including current prescription and over-the-counter treatments used for migraine. The CCI is a weighted index of the following comorbidities and sums the result: human immunodeficiency virus/acquired immunodeficiency syndrome, metastatic tumor, lymphoma, leukemia, any tumor, moderate/severe renal disease, hemiplegia, diabetes, mild liver disease, ulcer disease, connective tissue disease, chronic pulmonary disease, dementia, cerebrovascular disease, peripheral vascular disease, myocardial infarction, congestive heart failure, and diabetes with end-organ damage; scores are $1,2,3$, or 6 , and higher scores indicate greater comorbid burden on the patient. ${ }^{15}$

Respondents reported the number of headache days they had experienced in the previous 30 days, and HFDs were calculated by subtracting the number of headache days from 30. In addition, the number of work days missed and the number of household activity days missed due to migraine in the previous 6 months were collected. Migraine effect was 
assessed using the validated, 6-item Headache Impact Test (HIT-6) questionnaire, which measures the adverse effect of headache on social functioning, role functioning, vitality, cognitive functioning, and psychological distress. Scores range from 36 to 78, with higher scores reflecting greater effect of headaches (score: $\leq 49$, little or no impact; 50-55, some impact; 56-59, substantial impact; 60-78, severe impact). ${ }^{16}$

Work productivity was assessed using the validated, 6-item Work Productivity and Activity Impairment (WPAI) questionnaire, which measures the effect of one's general health on the extent of absenteeism (work time missed), presenteeism (impairment experienced while at work), overall work productivity loss (overall impairment estimate that is a combination of absenteeism and presenteeism), and activity impairment (impairment in regular daily activities other than work done for pay) during the previous 7 days. Only respondents who reported being employed full time or part time provided data for absenteeism, presenteeism, and overall work impairment. ${ }^{17}$ All respondents provided responses for activity impairment. WPAI data metrics are expressed as percentages, with higher values indicating greater impairment and less productivity. ${ }^{17}$

Estimated annualized indirect costs due to work productivity were calculated using the human capital method. ${ }^{18,19}$ Here we used median weekly income figures obtained from the 2014 Bureau of Labor Statistics and divided them by the length of a typical work week (40 hours) to first estimate an hourly rate (Supplementary Table 1, available in online article). ${ }^{20}$ The number of hours of work respondents missed the previous week because of absenteeism and presenteeism was obtained from the WPAI questionnaire. This estimate was multiplied by hourly rates to arrive at total lost wages for the week based on the assumption that the value of hours of lost work could be estimated by wages for that hour. These figures were then multiplied by the average number of work weeks in a year (assuming 50 work weeks per year) to obtain annual estimates.

To estimate the annualized direct costs from health care resource use, respondents reported the number of traditional health care provider (HCP) visits, emergency room visits, and hospitalizations during the previous 6 months due to their "own medical condition." That condition was migraine for participants in this study. The number of each type of visit reported by respondents was then multiplied by 2 to project to the annual number of visits and then multiplied by its average cost obtained from the Medical Expenditure Panel Survey (MEPS; Supplementary Table 1, available in online article). ${ }^{21}$ The MEPS is a large U.S. government survey study assessing health care resource use and cost. The unit cost within the database represents the payments made to HCPs according to the type of service, including both out-of-pocket payments by the patient and contributions from insurance. Full details of determination of costs within MEPS are available online (http://meps.ahrq.gov). Finally, figures were summed to a total annual direct cost value for each respondent. Many NHWS studies have been published using the same direct and indirect cost methodologies. ${ }^{22-26}$

\section{Statistical Analysis}

Multivariable analyses were conducted on the subgroup of respondents from the NHWS migraine module who reported experiencing $\geq 4$ headache days per month. The primary independent variable was the number of HFDs assessed as both continuous (number of HFDs in the previous 30 days) and categorical (0-10, 11-20, and 21-26 HFDs) measures. Both measures were used as predictors in separate generalized linear models (GLMs) specifying the appropriate distribution. Specifically, normal GLMs were used for HIT-6 data, whereas negative binomial distributions with log-link functions were used for WPAI data and costs because of the distribution of the WPAI data. Covariates included age, gender, ethnicity, and the CCI. Relevant covariates for inclusion in models were identified based on assessment of differences among patients with migraine with varying levels of HFDs categories with respect to demographics and health history variables. Chi-square tests and 1-way analysis of variance were used for categorical and continuous outcomes, respectively. Differences in sociodemographics (e.g., age, gender, ethnicity, education, income) and health characteristics (e.g., body mass index, depression) were not significant in bivariate analyses (Table 1). Moreover, we did not adjust for depression because it is likely in the causal pathway linking HFDs to outcomes of interest. We know that migraine is a risk factor for depression and that it influences many patient outcomes. ${ }^{27}$ However, age, gender, ethnicity, and the CCI were adjusted for, as is typical in burden of illness analyses.

This study was adequately powered (80\%) to detect a medium effect size (Cohen's $d=0.5$ ) between groups, which required 64 respondents per group; however, this study was underpowered to detect a small effect size (Cohen's d=0.2).

Results

\section{Characteristics of Study Sample}

A total of 97,503 respondents participated in the 2016 U.S. NHWS. Of those who self-reported a migraine diagnosis $(n=9,579), 933$ were randomly selected into the migraine module, and 372 of these respondents were identified as having $\geq 4$ headache days in the previous 30 days. For categorical analyses, respondents were further grouped according to the number of HFDs they experienced in the previous 30 days: $0-10$ HFDs, $\mathrm{n}=83$; 11-20 HFDs, $\mathrm{n}=116$; 21-26 HFDs, $\mathrm{n}=173$ (Supplementary Figure 1, available in online article). Respondents, on average, were aged 40.3 years and had a CCI value of 0.69 . They were mainly female $(80.9 \%)$ and white $(61.6 \%)$, and most had less than a 4-year college degree (62.4\%; Table 1). The most commonly used treatments were over-thecounter medications or herbal products (64.5\%). 
TABLE 1 Demographics and Health Characteristics of Respondents with Migraine by Headache-Free Days Category Among Those with $\geq 4$ Headache Days in the Previous 30 Days

\begin{tabular}{|c|c|c|c|c|c|c|}
\hline \multirow[b]{3}{*}{ Age, years, mean (SD) } & \multicolumn{3}{|c|}{ Chronic Migraine } & \multicolumn{3}{|c|}{ Episodic Migraine } \\
\hline & $\begin{array}{c}0-10 \text { HFDs } \\
n=83\end{array}$ & $\begin{array}{c}11-15 \text { HFDs } \\
n=50\end{array}$ & \multirow{2}{*}{$\frac{P \text { Value }^{\mathrm{a}}}{0.418}$} & $\begin{array}{c}16-20 \text { HFDs } \\
n=66\end{array}$ & $\begin{array}{c}21-26 \text { HFDs }^{b} \\
\mathrm{n}=173\end{array}$ & \multirow{2}{*}{$\frac{P \text { Value }}{0.201}$} \\
\hline & $42.8 \quad(14.4)$ & $40.8 \quad(12.9)$ & & $41.1 \quad(13.1)$ & $38.7 \quad(12.7)$ & \\
\hline CCI score, mean (SD) & $0.7 \quad(1.1)$ & $0.7 \quad(1.1)$ & 0.856 & $0.9 \quad(1.6)$ & $0.6 \quad(1.4)$ & 0.088 \\
\hline Women, n (\%) & $69 \quad(83.1)$ & $43 \quad(86.0)$ & 0.660 & $50 \quad(75.8)$ & $139 \quad(80.3)$ & 0.435 \\
\hline \multicolumn{7}{|l|}{ Race/ethnicity, n (\%) } \\
\hline White/non-Hispanic & $59 \quad(71.1)$ & $31 \quad(62.0)$ & 0.123 & $40 \quad(60.6)$ & $99 \quad(57.2)$ & 0.722 \\
\hline Black/non-Hispanic & $1 \quad(1.2)$ & $5 \quad(10.0)$ & - & $7 \quad(10.6)$ & $13 \quad(7.5)$ & - \\
\hline Hispanic & $9 \quad(10.8)$ & $6 \quad(12.0)$ & - & $7 \quad(10.6)$ & $25 \quad(14.5)$ & - \\
\hline Other ethnicity & $14 \quad(16.9)$ & $8 \quad(16.0)$ & - & $12(18.2)$ & $36 \quad(20.8)$ & - \\
\hline \multicolumn{7}{|l|}{ Education, n (\%) } \\
\hline$<4$-year college degree & $52 \quad(62.7)$ & $31 \quad(62.0)$ & 0.940 & $43 \quad(65.2)$ & $106 \quad(61.3)$ & 0.580 \\
\hline 4-year college degree or higher & $31 \quad(37.3)$ & $19 \quad(38.0)$ & - & $23 \quad(34.8)$ & $67 \quad(38.7)$ & - \\
\hline \multicolumn{7}{|l|}{ Annual household income, n (\%) } \\
\hline$<\$ 25,000$ & $19 \quad(22.9)$ & $11 \quad(22.0)$ & 0.976 & $9 \quad(13.6)$ & $40 \quad(23.1)$ & 0.438 \\
\hline$\$ 25,000-\$ 50,000$ & $28 \quad(33.7)$ & $17(34.0)$ & - & $23 \quad(34.8)$ & $44 \quad(25.4)$ & - \\
\hline$\$ 50,000-\$ 75,000$ & $11 \quad(13.3)$ & $5 \quad(10.0)$ & - & $10 \quad(15.2)$ & $26 \quad(15.0)$ & - \\
\hline$\geq \$ 75,000$ & $20 \quad(24.1)$ & $14 \quad(28.0)$ & - & $21 \quad(31.8)$ & $57 \quad(32.9)$ & - \\
\hline Declined to answer & $5 \quad(6.0)$ & $3 \quad(6.0)$ & - & $3 \quad(4.5)$ & $6 \quad(3.5)$ & - \\
\hline Married/living with partner, n (\%) & $47 \quad(56.6)$ & $26 \quad(52.0)$ & 0.604 & $36 \quad(54.5)$ & $100 \quad(57.8)$ & 0.649 \\
\hline \multicolumn{7}{|l|}{ BMI category, n (\%) } \\
\hline Underweight (range: BMI < 18.5) & $4 \quad(4.8)$ & $3 \quad(6.0)$ & 0.995 & $1 \quad(1.5)$ & $5 \quad(2.9)$ & 0.990 \\
\hline Normal weight (range: BMI 18.5 to $<25.0$ ) & $22(26.5)$ & $12(24.0)$ & - & $23 \quad(34.8)$ & $55 \quad(31.8)$ & - \\
\hline Overweight (range: BMI 25.0 to $<30.0$ ) & $25 \quad(30.1)$ & $15(30.0)$ & - & $18 \quad(27.3)$ & $48 \quad(27.7)$ & - \\
\hline Obese (range: $\mathrm{BMI} \geq 30.0$ ) & $30 \quad(36.1)$ & $19 \quad(38.0)$ & - & $23 \quad(34.8)$ & $64 \quad(37.0)$ & - \\
\hline Unknown BMI & $2 \quad(2.4)$ & $1 \quad(2.0)$ & - & $1 \quad(1.5)$ & $1 \quad(0.6)$ & - \\
\hline \multicolumn{7}{|l|}{ Smoking status, n (\%) } \\
\hline Current smoker & $18 \quad(21.7)$ & $12(24.0)$ & 0.927 & $18 \quad(27.3)$ & $37 \quad(21.4)$ & 0.230 \\
\hline Former smoker & $22(26.5)$ & $12(24.0)$ & - & $18 \quad(27.3)$ & $36 \quad(20.8)$ & - \\
\hline Never a smoker & $43 \quad(51.8)$ & $26 \quad(52.0)$ & - & $30 \quad(45.5)$ & $100 \quad(57.8)$ & - \\
\hline Drinks alcohol, n (\%) & $52 \quad(62.7)$ & $32(64.0)$ & 0.876 & $42 \quad(63.6)$ & $131 \quad(75.7)$ & 0.062 \\
\hline \multicolumn{7}{|l|}{ Comorbid diagnoses, n (\%) } \\
\hline Depression & $54 \quad(65.1)$ & $26 \quad(52.0)$ & 0.136 & $37 \quad(56.1)$ & $90 \quad(52.0)$ & 0.576 \\
\hline Generalized anxiety disorder & $19 \quad(22.9)$ & $7 \quad(14.0)$ & 0.210 & $8 \quad(12.1)$ & $24 \quad(13.9)$ & 0.722 \\
\hline Sleep apnea & $12 \quad(14.5)$ & $3 \quad(6.0)$ & 0.135 & $4 \quad(6.1)$ & $14 \quad(8.1)$ & 0.595 \\
\hline HIT-6 total score, mean (SD) & $66.3 \quad(5.6)$ & $(5.6)$ & 0.689 & $(5.4)$ & $(6.8)$ & 0.058 \\
\hline \multicolumn{7}{|l|}{ Work productivity impairment, mean (SD) } \\
\hline Absenteeism, \%c & $14.9 \quad(25.3)$ & $13.6 \quad(27.7)$ & 0.848 & $9.3(13.7)$ & $8.8(16.8)$ & 0.875 \\
\hline Presenteeism, \%d & $43.6 \quad(32.5)$ & $33.0 \quad(30.7)$ & 0.219 & $33.5 \quad(28.4)$ & $30.5 \quad(27.0)$ & 0.574 \\
\hline Overall work impairment, $\%^{c}$ & $48.0 \quad(35.5)$ & $40.2 \quad(35.4)$ & 0.399 & $37.5 \quad(29.4)$ & $34.5(29.5)$ & 0.601 \\
\hline \multicolumn{7}{|c|}{ Health care resource use in past 6 months, mean (SD) } \\
\hline HCP visits & $(6.5)$ & $(8.0)$ & 0.925 & $(5.5)$ & $7.1 \quad(11.2)$ & 0.392 \\
\hline ER visits & $(1.1)$ & $(2.9)$ & 0.419 & $(1.1)$ & $0.5 \quad(1.3)$ & 0.909 \\
\hline Hospitalizations & $(0.6)$ & $(1.4)$ & 0.499 & $(0.6)$ & $(0.8)$ & 0.353 \\
\hline
\end{tabular}

a Based on 1-way ANOVA for age, CCI, HIT-6, and work productivity impairment. Based on chi-square test for all other characteristics.

${ }^{b}$ Because eligible participants must have had $\geq 4$ headache days per month, no patient could have $>26$ HFDs in the previous 30-day period.

conly calculated for employed respondents; 0-10 HFDs, $n=37$; 11-15 HFDs, $n=25$; $16-20$ HFDs, $n=37$; 21-26 HFDs, $n=95$.

'Only calculated for employed respondents; 0-10 HFDs, $n=36$; 11-15 HFDs, $n=23$; 16-20 HFDs, $n=37$; 21-26 HFDs, $n=95$.

ANOVA = analysis of variance; $B M I=$ body mass index; $C C I=$ Charlson Comorbidity Index; $E R=$ emergency room; $H C P=$ health care provider; $H F D=$ headache-free day; HIT-6 =6-item Headache Impact Test; $S D=$ standard deviation. 


\begin{tabular}{|c|c|c|c|}
\hline $\begin{array}{c}\text { TABLE } 2 \text { Summary of Regression Results for the Numb } \\
\text { Predicting Outcomes Among Respondents w } \\
\text { Previous } 30 \text { Days }\end{array}$ & $\begin{array}{l}\text { of Headache-Fre } \\
\text { Migraine Experi }\end{array}$ & $\begin{array}{l}\text { ays as a Con } \\
\text { ng } \geq 4 \text { Head }\end{array}$ & $\begin{array}{l}\text { Variable } \\
\text { ys in the }\end{array}$ \\
\hline Outcome & Beta Coefficient (SE) & RR (95\% CI) & $P$ Value ${ }^{a}$ \\
\hline HIT-6 total score & $-0.15 \quad(0.04)$ & - & $<0.001$ \\
\hline Days of work missed in the past 6 months due to migraine & $-0.05 \quad(0.02)$ & $0.95 \quad(0.92-0.99)$ & 0.012 \\
\hline Days of household activities missed in the previous 6 months due to migraine & $-0.06 \quad(0.01)$ & $(0.93-0.97)$ & $<0.001$ \\
\hline Indirect $\operatorname{costs}^{\mathrm{b}}$ & $-0.04 \quad(0.02)$ & $(0.92-1.00)$ & 0.38 \\
\hline Absenteeism & $-0.03 \quad(0.02)$ & $(0.94-1.01)$ & 0.108 \\
\hline Presenteeism & $-0.01 \quad(0.01)$ & $(0.97-1.01)$ & 0.219 \\
\hline Overall work impairment & $-0.01 \quad(0.01)$ & $(0.97-1.01)$ & 0.207 \\
\hline Direct costs ${ }^{c}$ & $0.01 \quad(0.02)$ & $(0.97-1.04)$ & 0.710 \\
\hline HCP visits & $0.00 \quad(0.01)$ & $(0.99-1.02)$ & 0.741 \\
\hline ER visits & $-0.01 \quad(0.02)$ & $0.99 \quad(0.95-1.03)$ & 0.502 \\
\hline Hospitalizations ${ }^{\mathrm{d}}$ & - & - & - \\
\hline 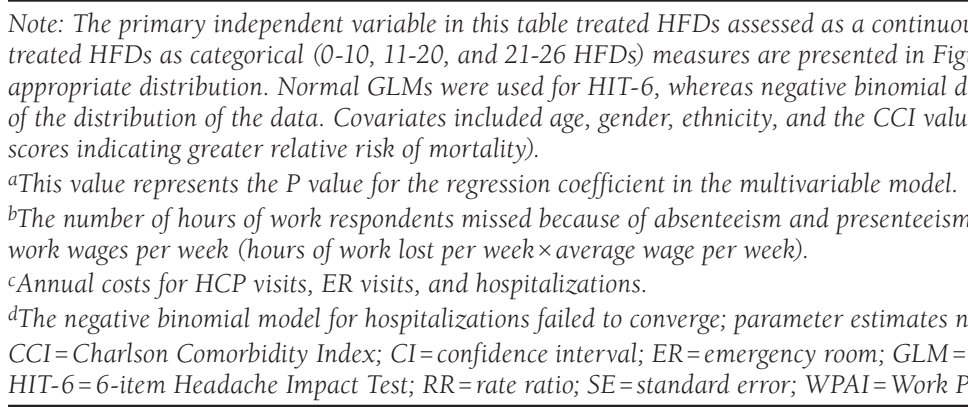 & $\begin{array}{l}\text { easure (number of HFDs } \\
\text { 1-3. Both measures were } \\
\text { butions with log-link funct } \\
\text { weighted index of comorb } \\
\text { sobtained from the WPAI } \\
\text { vailable. } \\
\text { eralized linear model; } \mathrm{HCH}\end{array}$ & $\begin{array}{l}\text { he previous } 30 \text { days). } \\
\text { d as predictors in sepa } \\
\text { is were used for WPAI } \\
\text { ies with scores of } 1,2 \text {, } \\
\text { estionnaire and entere } \\
\text { health care provider; }\end{array}$ & $\begin{array}{l}\text { e models that } \\
\text { Ms specifying th } \\
\text { hd costs because } \\
\text { with higher } \\
\text { model as lost } \\
\text { eadache-free day }\end{array}$ \\
\hline
\end{tabular}

\section{Effect of Migraine on Daily Life}

Each HFD was associated with a 0.15 (regression coefficient)point reduction in HIT-6 scores (Table 2). Furthermore, each HFD was associated with a rate ratio (RR) of 0.95 for the days of work missed due to migraine; in other words, as HFDs increased, days of work significantly decreased. Similarly, for the days of household activities missed, the RR was 0.95 for each HFD (Table 2). This translates to a 5\% reduction in both the number of work days missed and the number of days of household activities missed for each incremental increase in HFD.

Respondents experiencing 21-26 HFDs in the previous 30 days had significantly lower HIT-6 total scores than those with 0-10 and those with 11-20 HFDs during that same time period (Figure 1). Similarly, compared with respondents with 0-10 HFDs and respondents with 11-20 HFDs, those experiencing 21-26 HFDs missed significantly fewer work days due to migraine (Figure 2). In assessing household activity days, the effect of migraine was greatest on respondents experiencing only 0-10 HFDs as they missed significantly more household activity days than those experiencing 11-20 and those experiencing 21-26 HFDs (Figure 2).

\section{Cost Savings Associated with HFDs}

Each HFD was associated with an RR of 0.96 for the indirect costs of overall work productivity loss. This translates to a $4 \%$ reduction in indirect costs for each additional HFD
(Table 2). Furthermore, increasing the number of HFDs from 0-10 HFDs to 21-26 HFDs was associated with significantly lower annual indirect costs (Figure 3). Annual direct costs due to health care resource utilization were similar across different HFD categories (Figure 3).

\section{Discussion}

The burden of disease associated with migraine, including the differences between EM and CM, have been well documented. ${ }^{1,2,6-8,28}$ The aim of this analysis of the 2016 NHWS examining respondents experiencing migraine with $\geq 4$ headache days per month was to assess the incremental reduction in burden associated with each additional HFD. Our study shows that an increase in the number of HFDs experienced by an individual is associated with improved functional status and well-being. Specifically, each HFD was associated with decreased headache-related adverse effect (as measured by a 0.15 -point decrease in the HIT-6) and a 5\% reduction in days of work and household activities missed due to migraine. Though this 5\% decrement may seem modest, it is per HFD. A treatment that increased HFDs per month by 5 could produce a 0.75 -point decrease in the HIT- 6 and a substantial $25 \%$ reduction in days of work or household activities missed. By extension, a treatment that increased HFDs per month by 15 could produce a clinically significant 2.3 -point decrease in the HIT-6. ${ }^{29}$ 

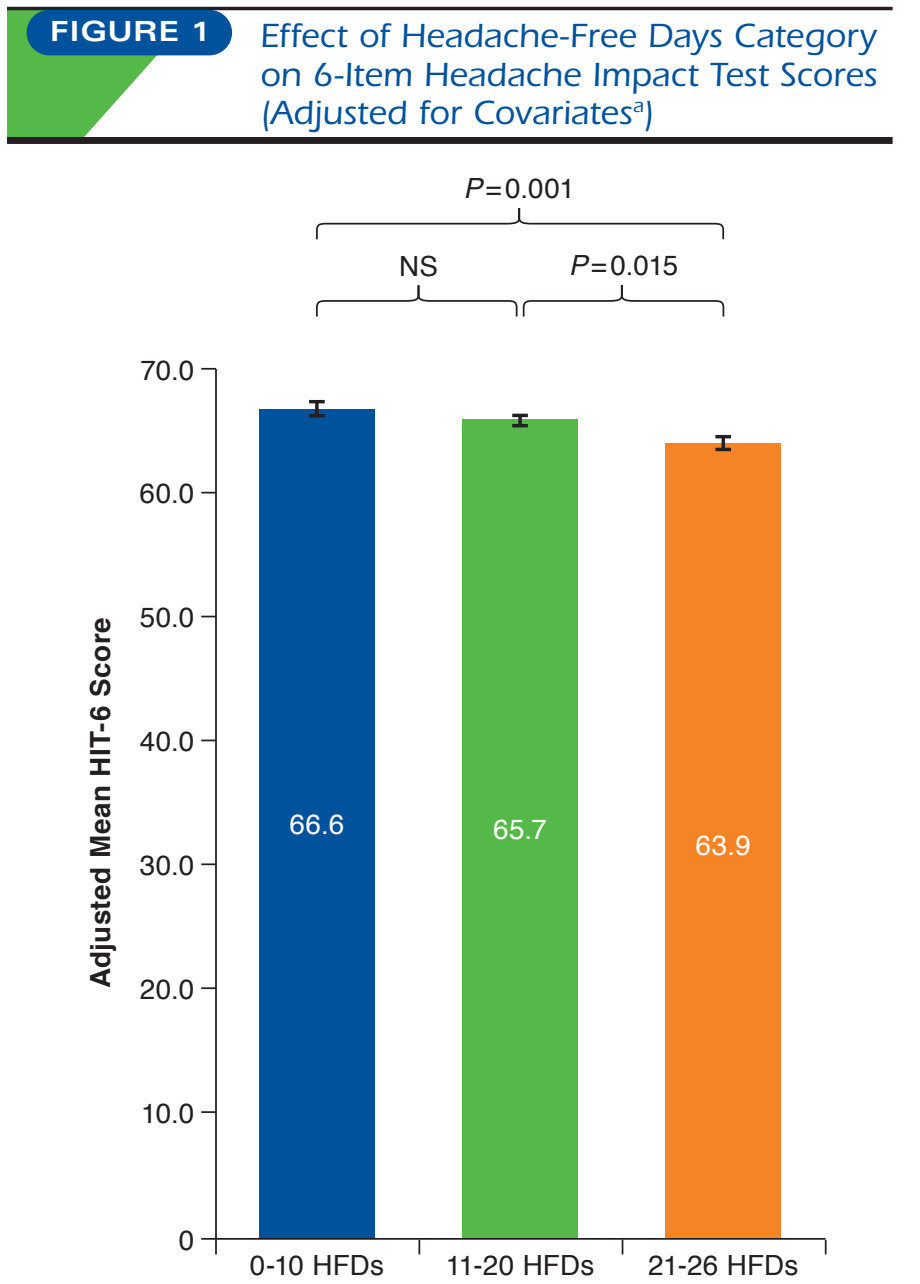

${ }^{a}$ Covariates included age, gender, ethnicity, and Charlson Comorbidity Index score. Error bars indicate mean \pm standard error.

HFD = headache-free day; HIT-6 =6-item Headache Impact Test; NS= not significant.

A similar study conducted by Doane et al. (2019) investigated the associations between the number of HFDs and HRQOL (Short-Form 12-Item Survey Instrument, version 2 physical and mental component summary scores and EuroQoL-5D scores), work productivity (WPAI questionnaire), and health care resource utilization in patients with migraine in the European Union Five (France, Germany, Italy, Spain, and the United Kingdom) using survey responses from the 2017 NHWS. ${ }^{30}$ Like the current study, patients with migraine had $\geq 4$ headache days in the previous month; however, the study population was much larger due to combining 4 countries $(\mathrm{N}=1,569)$. Multivariable analyses adjusted for the following covariates: sex, country, employment status, marital status, household income, education, body mass index, alcohol use, exercise, age in years, and CCI. Similar results were observed in terms of associations between HFDs and WPAI: the RR for each HFD was 0.961 for the days of work missed due to migraine and 0.982 for the days of household activities missed, translating to a $3.9 \%$ reduction in days of work and $1.8 \%$ reduction in days of household activities missed due to migraine. This study also showed a decrease in resource utilization with increasing HFDs. With an increase of 1 HFD, decreases of $1.0 \%$ and $4.7 \%$ were anticipated over 6 months in HCP and neurologist visits, respectively. ${ }^{30}$ Results of the current study in a U.S. population of patients with migraine support and expand on this previous study in a European population, emphasizing the benefits of increasing HFDs across multiple different populations of patients.

Improvements were also seen in economic terms, as each HFD was associated with a $4 \%$ reduction in indirect costs, although the reduction was not statistically significant. This suggests that treatments that increase HFDs may help decrease the annual indirect costs by reducing the number of work days missed due to migraine. For example, a treatment that resulted in a 5-day increase in HFDs would be associated with an estimated $20 \%$ reduction in indirect costs. The estimate was based on hourly rates multiplied by lost days to arrive at total lost wages for the week. This approach could lead to underestimates if one worker's absence influences the productivity of other workers. It could lead to overestimates if a worker makes up for his or her absence or if coworkers make up for an absence..$^{31-33}$ It is possible this assumption does not correctly estimate the value of hours of lost work based on wages for that hour alone. There is also the potential for overestimation of costs associated with work days missed since results from 1 week of lost wages were extrapolated to a year; however, this approach, the human capital approach, has been widely used in calculating cost of illness. ${ }^{18,19}$

Estimated annual direct costs were similar for respondents in all categories of HFDs. One possible explanation is that we did not fully ascertain direct medical costs. Our estimates did not include the use of diagnostic technology or prescription and over-the-counter medications, for example. Blumenfeld et al. (2011) found differences in health care resource utilization along the migraine continuum; however, the Blumenfeld et al. study did not exclude individuals with $<4$ headache days and compared only 2 groups (EM and CM), potentially accounting for the different result. ${ }^{6}$

To be included in this study, respondents had to have experienced at least 4 headache days in the previous 30 days; therefore, the study population was made up of individuals eligible for preventive treatment, ${ }^{13}$ including those with $\mathrm{CM}$ and those at an elevated risk for developing CM. ${ }^{9}$ Only a fraction of those eligible to take preventive therapy actually did so. ${ }^{13}$ If these cross-sectional results demonstrating that each HFD provides a benefit are generalizable, physicians and their patients may want to reassess how they treat and manage migraine to 


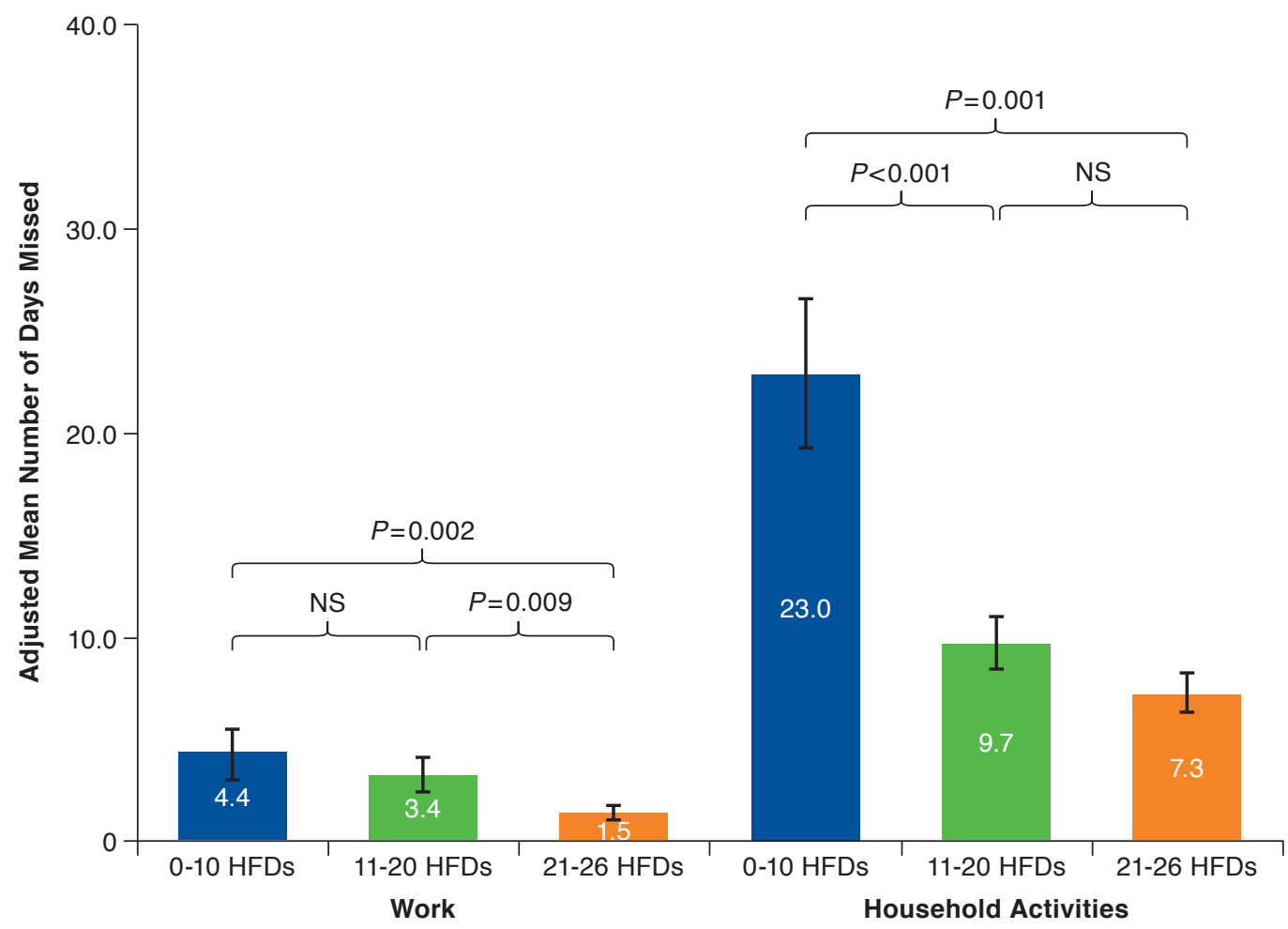

aCovariates included age, gender, ethnicity, and Charlson Comorbidity Index score. Error bars indicate mean \pm standard error. HFD = headache-free day; NS = not significant.

minimize the burden of the disease and accrue the benefits of HFDs. The inclusion of only persons with self-reported migraine who have 4 or more headache days per month may limit the generalizability of our findings.

\section{Limitations}

This study was based on data from the cross-sectional 2016 NHWS and has several limitations. First, as previously noted, the sample is not fully generalizable to people with migraine. For example, the sample comprised individuals who were recruited through a web-based consumer panel and thus does not include those without access to or comfort with online, internet-based surveys. In addition, the sample included only those with self-reported diagnosis of migraine by an HCP. This group is likely enriched for people with more severe migraine and better access to medical care.

Second, we assumed that the value of an hour of missed work is measured by wages for that hour of work. This assumption has been challenged. ${ }^{33}$ Third, although multivariable analyses were used to adjust for several confounding factors, there are other measured and unmeasured variables that were not taken into account. Nevertheless, no significant differences in sociodemographic and health characteristics between HFD groups were observed in bivariate analyses.

Fourth, this is a cross-sectional and not a longitudinal study, so information on temporal sequence is not available, limiting causal inferences. Fifth, the self-reported nature of the NHWS is also associated with potential biases such as inaccurate recall and false reporting (whether intentional or unintentional). For example, migraine status was self-reported as opposed to being based on medical records or direct patient interviews. Headache days were also self-reported and used to calculate HFDs. In many interviews, headache days are underreported, which could lead to a potential measurement error in our estimates of HFDs.

Furthermore, because individuals with $<4$ headache days per month were excluded from this study, inferences about this population cannot be drawn. In this study, we estimated monthly headache days, not monthly migraine days. However, there is some evidence that in people with migraine, 


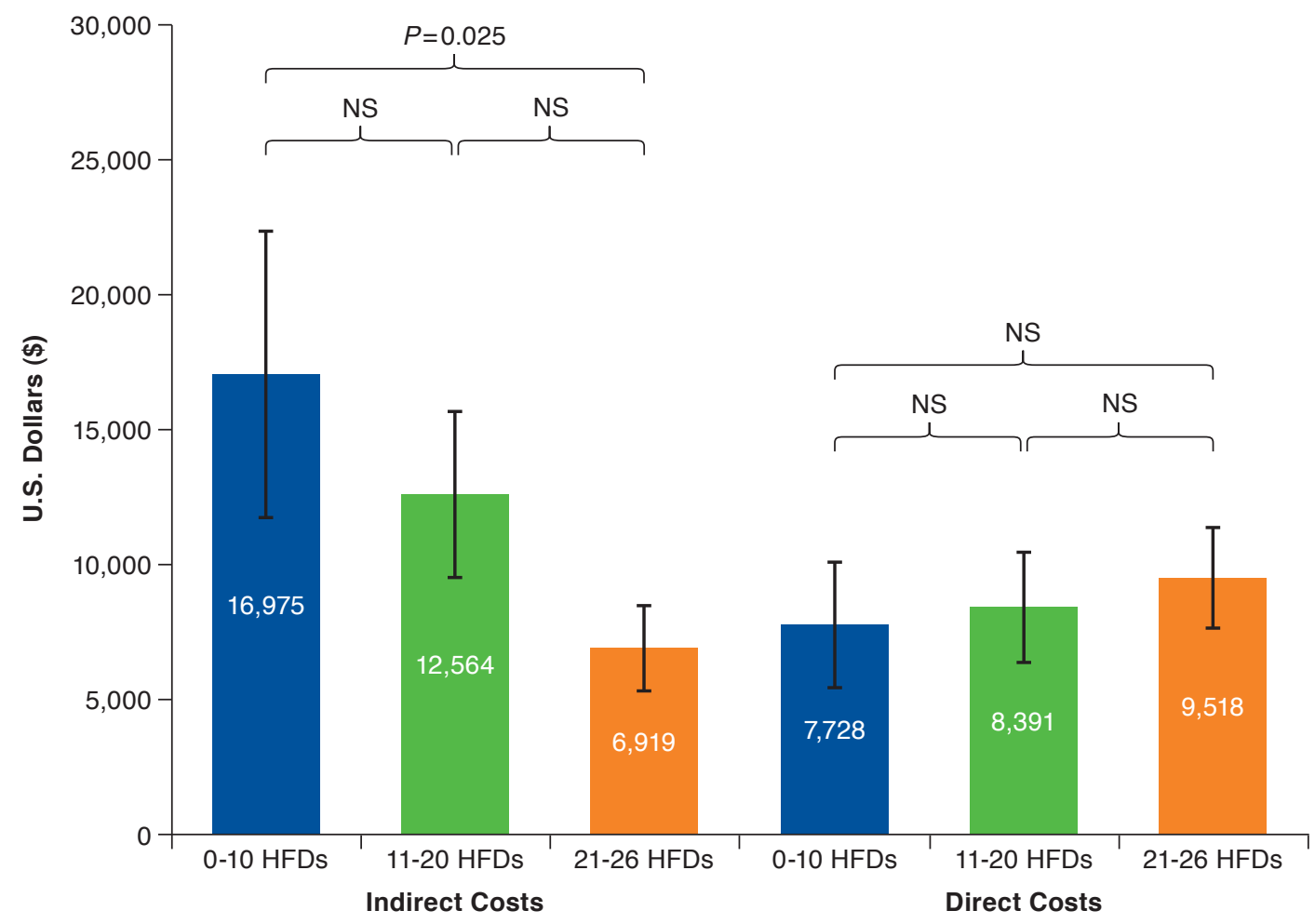

${ }^{a}$ Covariates included age, gender, ethnicity, and Charlson Comorbidity Index score. Error bars indicate mean \pm standard error. $H F D=$ headache-free day; $N S=$ not significant .

phenotypic tension-type headache has a migraine mechanism, ${ }^{34,35}$ and there is also evidence that most headaches meet criteria for migraine. ${ }^{36}$ It should be noted that tension and cluster headaches were not specifically assessed in this study.

Finally, the sample size was relatively small $(\mathrm{N}=372)$, particularly when further divided for categorical analyses based on the number of HFDs.

Despite these limitations, this study provides a perspective on the burden of migraine by describing it in terms closely aligned to the way individuals experience the disease on a daily level. These results reinforce the unmet need for effective, safe, well-tolerated migraine treatment options.

\section{Conclusions}

This study demonstrates that each increase in HFDs is associated with a reduction in headache-related adverse effect on functional status and well-being, a reduction in days of work and household activities missed due to migraine, and a reduction in indirect costs. These findings suggest that increasing the number of HFDs experienced by individuals may reduce the burden and cost associated with migraine.

\section{Authors}

RICHARD B. LIPTON, MD, Albert Einstein College of Medicine and Montefiore Medical Center, Bronx, New York. LULU LEE, PhD, Kantar, San Mateo, California. NICOLAS P. SAIKALI, MD, FAHS, Dent Neurologic Institute, Buffalo, New York. JVAWNNA BELL, MPH, MBA, and JOSHUA M. COHEN, MD, MPH, FAHS, Teva Branded Pharmaceutical Products RED, West Chester, Pennsylvania.

AUTHOR CORRESPONDENCE: Richard B. Lipton, MD, Albert Einstein College of Medicine, 1300 Morris Park Ave., \#3, Bronx, NY 10461. Tel.: 718.430.3886; Email: richard.lipton@einsteinmed.org. 
serves as consultant, advisory board member, or has received honoraria from the American Academy of Neurology, Alder, Allergan, American Headache Society, Amgen, Autonomic Technologies, Avanir, Biohaven, Biovision, Boston Scientific, Dr. Reddy's, electroCore, Eli Lilly, eNeura Therapeutics, GlaxoSmithKline, Merck, Pernix, Pfizer, Supernus, Teva, Trigemina, Vector, and Vedanta. Saikali serves on the advisory board and as speaker for Allergan, Amgen, Promius, Supernus, and Teva Pharmaceuticals. He serves as a speaker for Assertio, Avanir, Cefaly, Egalet, Eli Lilly, Gammacore, and Pernix.

This study has been presented as a poster at the American Academy of Neurology 2018 Annual Meeting, April 21-27, 2018, in Los Angeles, CA; Headache Update 2017, July 13-16, 2017, in Lake Buena Vista, FL; and the American Headache Society 2017 Annual Meeting, June 8-11, 2017, in Boston, MA.

\section{ACKNOWLEDGMENTS}

The authors thank Lindsay Tannenholz, PhD (Chameleon Communications International with funding from Teva Pharmaceutical Industries) for editorial assistance in the preparation of this report.

\section{REFERENCES}

1. GBD 2016 Headache Collaborators. Global, regional, and national burden of migraine and tension-type headache, 1990-2016: a systematic analysis for the Global Burden of Disease Study 2016. Lancet Neurol. 2018;17(11):954-76.

2. Buse DC, Rupnow MF, Lipton RB. Assessing and managing all aspects of migraine: migraine attacks, migraine-related functional impairment, common comorbidities, and quality of life. Mayo Clin Proc. 2009;84(5):422-35

3. Gooch CL, Pracht E, Borenstein AR. The burden of neurological disease in the United States: a summary report and call to action. Ann Neurol. 2017:81(4):479-84

4. Katsarava Z, Buse DC, Manack AN, Lipton RB. Defining the differences between episodic migraine and chronic migraine. Curr Pain Headache Rep. 2012;16(1):86-92

5. Lipton RB, Silberstein SD. Episodic and chronic migraine headache: breaking down barriers to optimal treatment and prevention. Headache. 2015;55(Suppl 2):103-22

6. Blumenfeld AM, Varon SF, Wilcox TK, et al. Disability, HRQoL and resource use among chronic and episodic migraineurs: results from the International Burden of Migraine Study (IBMS). Cephalalgia. 2011;31(3):301-15.

7. Bigal ME, Serrano D, Reed M, Lipton RB. Chronic migraine in the population: burden, diagnosis, and satisfaction with treatment. Neurology. 2008;71(8):559-66.

8. Buse DC, Manack AN, Fanning KM, et al. Chronic migraine prevalence, disability, and sociodemographic factors: results from the American Migraine Prevalence and Prevention Study. Headache. 2012;52(10):1456-70.

9. Aurora SK, Brin MF. Chronic migraine: an update on physiology, imaging, and the mechanism of action of two available pharmacologic therapies. Headache. 2017;57(1):109-25.

10. Lipton RB, Fanning KM, Serrano D, Reed ML, Cady R, Buse DC Ineffective acute treatment of episodic migraine is associated with new-onset chronic migraine. Neurology. 2015;84(7):688-95.

11. Buse DC, Greisman JD, Baigi K, Lipton RB. Migraine progression: a systematic review. Headache. 2019;59(3):306-38.

12. American Headache Society. The American Headache Society position statement on integrating new migraine treatments into clinical practice. Headache. 2019;59(1):1-18.

13. Lipton RB, Bigal ME, Diamond M, et al. Migraine prevalence, disease burden, and the need for preventive therapy. Neurology. 2007;68(5):343-49. 14. Zikmund-Fisher BJ, Couper MP, Singer E, et al. The DECISIONS study: a nationwide survey of United States adults regarding 9 common medical decisions. Med Decis Making. 2010;30(5 Suppl):20S-34S.

15. Charlson ME, Pompei P, Ales KL, MacKenzie CR. A new method of classifying prognostic comorbidity in longitudinal studies: development and validation. J Chronic Dis. 1987;40(5):373-83.
16. Yang M, Rendas-Baum R, Varon SF, Kosinski M. Validation of the Headache Impact Test (HIT-6 $6^{\mathrm{TM}}$ ) across episodic and chronic migraine. Cephalalgia. 2011;31(3):357-67.

17. Reilly MC, Zbrozek AS, Dukes EM. The validity and reproducibility of a work productivity and activity impairment instrument. Pharmacoeconomics. 1993;4(5):353-65

18. Lofland JH, Pizzi L, Frick KD. A review of health-related workplace productivity loss instruments. Pharmacoeconomics. 2004;22(3):165-84.

19. Xie F, Thumboo J, Fong KY, et al. A study on indirect and intangible costs for patients with knee osteoarthritis in Singapore. Value Health. 2008;11(Suppl 1):S84-S90

20. U.S. Bureau of Labor Statistics. Highlights of women's earnings in 2014 November 2015. Available at: https://www.bls.gov/opub/reports/womensearnings/archive/highlights-of-womens-earnings-in-2014.pdf. Accessed June 18,2020

21. Agency for Healthcare Research and Quality. Mean expenditure per event by age groups and event type, United States, 2014. Medical Expenditure Panel Survey. Available at: https://meps.ahrq.gov/mepstrends/ hc_use/. Generated interactively: June 30, 2020.

22. Flores NM, Villa KF, Black J, Chervin RD, Witt EA. The humanistic and economic burden of narcolepsy. J Clin Sleep Med. 2016;12(3):401-07. 23. Dibonaventura MD, Wagner JS, Alvir J, Whiteley J. Depression, quality of life, work productivity, resource use, and costs among women experiencing menopause and hot flashes: a cross-sectional study. Prim Care Companion CNS Disord. 2012;14(6):PCC.12m01410.

24. Gupta S, Isherwood G, Jones K, Van Impe K. Productivity loss and resource utilization, and associated indirect and direct costs in individuals providing care for adults with schizophrenia in the EU5. Clinicoecon Outcomes Res. 2015;7:593-602.

25. Moline M, DiBonaventura Md, Shah D, Ben-Joseph R. Impact of middleof-the-night awakenings on health status, activity impairment, and costs. Nat Sci Sleep. 2014;6:101-11.

26. El Khoury AC, Vietri J, Prajapati G. The burden of untreated hepatitis C virus infection: a US patients' perspective. Dig Dis Sci. 2012;57(11):2995-3003

27. Breslau N, Lipton RB, Stewart WF, Schultz LR, Welch KM. Comorbidity of migraine and depression: investigating potential etiology and prognosis. Neurology. 2003;60(8):1308-12.

28. Hawkins K, Wang S, Rupnow MF. Indirect cost burden of migraine in the United States. J Occup Environ Med. 2007;49(4):368-74.

29. Coeytaux RR, Kaufman JS, Chao R, Mann JD, Devellis RF. Four methods of estimating the minimal important difference score were compared to establish a clinically significant change in Headache Impact Test. J Clin Epidemiol. 2006;59(4):374-80.

30. Doane MJ, Gupta S, Vo P, Laflamme AK, Fang J. Associations between headache-free days and patient-reported outcomes among migraine patients: a cross-sectional analysis of survey data in Europe. Pain Ther. 2019;8(2):203-16

31. Lensberg BR, Drummond MF, Danchenko N, Despiegel N, Francois C. Challenges in measuring and valuing productivity costs, and their relevance in mood disorders. Clinicoecon Outcomes Res. 2013;5:565-73.

32. Stromberg C, Aboagye E, Hagberg J, Bergstrom G, Lohela-Karlsson M. Estimating the effect and economic impact of absenteeism, presenteeism, and work environment-related problems on reductions in productivity from a managerial perspective. Value Health. 2017;20(8):1058-64.

33. Nicholson S, Pauly MV, Polsky D, Sharda C, Szrek H, Berger ML. Measuring the effects of work loss on productivity with team production. Health Econ. 2006;15(2):111-23.

34. Lipton RB, Cady RK, Stewart WF, Wilks K, Hall C. Diagnostic lessons from the Spectrum study. Neurology. 2002;58(9 Suppl 6):S27-S31.

35. Lipton RB, Stewart WF, Cady R, et al. 2000 Wolfe Award. Sumatriptan for the range of headaches in migraine sufferers: results of the Spectrum Study. Headache. 2000;40(10):783-91.

36. Blumenthal HJ, Weisz MA, Kelly KM, Mayer RL, Blonsky J. Treatment of primary headache in the emergency department. Headache. 2003;43(10):1026-31. 